\title{
Testing of NASA LaRC materials under MISSE 6 and MISSE 7 missions
}

\author{
Narasimha S. Prasad ${ }^{*}$ \\ NASA Langley Research Center, 5 N. Dryden St., MS 468, Hampton VA, 23681
}

\begin{abstract}
The objective of the Materials International Space Station Experiment (MISSE) is to study the performance of novel materials when subjected to the synergistic effects of the harsh space environment for several months. MISSE missions provide an opportunity for developing space qualifiable materials. Two lasers and a few optical components from NASA Lnagley Research Center (LaRC) were included in the MISSE 6 mission for long term exposure. MISSE 6 items were characterized and packed inside a ruggedized Passive Experiment Container (PEC) that resembles a suitcase. The PEC was tested for survivability due to launch conditions. MISSE 6 was transported to the international Space Station (ISS) via STS 123 on March 11. 2008. The astronauts successfully attached the PEC to external handrails of the ISS and opened the PEC for long term exposure to the space environment. The current plan is to bring the MISSE 6 PEC back to the Earth via STS 128 mission scheduled for launch in August 2009. Currently, preparations for launching the MISSE 7 mission are progressing. Laser and lidar components assembled on a flight-worthy platform are included from NASA LaRC. MISSE 7 launch is scheduled to be launched on STS 129 mission. This paper will briefly review recent efforts on MISSE 6 and MISSE 7 missions at NASA Langley Research Center (LaRC).
\end{abstract}

Keywords: MISSE 6, MISSE 7, Space Qualification, Laser components, International Space Station, STS-123.

\section{INTRODUCTION}

The goal of MISSE program is to evaluate the performance, stability, and long-term survivability of materials and components planned for use by NASA, Department of Defense (DOD), other federal agencies and private entities on future Low Earth Orbit (LEO), synchronous orbit, and interplanetary space missions. The study of combined effects of radiation, temperature cycling, ultraviolet (UV) light and atomic oxygen in space environment due to long term exposures will help in developing space qualifiable elements for future space missions. In-situ space testing is critical since it is difficult and not economical to conduct these studies inside simulated terrestrial facilities. The development of new generations of materials will allow our nation to continue maintain technological superiority related to space endeavors.

NASA's Langley Research Center (LaRC), Hampton, Virginia has managed six MISSE projects. Other NASA Centers participating in this project include Glenn Research Center, Cleveland; Goddard Space Flight Center, Greenbelt, Maryland, Johnson Space Center, Houston, Texas, the Jet Propulsion Laboratory, Pasadena, California, and Marshall Space Flight Center, Huntsville, Alabama. Several DOD and industry partners including the Boeing Company have been involved with this effort. MISSE program is a direct successor of the Mir Environmental Effects Payloads (MEEP) that were attached for over a year to the Mir Docking Module of the space station Mir between shuttle flights STS-76 and STS-86 ${ }^{1}$.

\section{AN OVERVIEW OF THE MISSE PROGRAM}

MISSE is a series of experiments and so far, five MISSE missions have been successfully completed. More than 1500 samples have been tested on the MISSE project. Samples include chemicals, sensor devices, opto-mechanical elements, polymers, coatings, and biological materials and species, composites. The MISSE project has also provided educational opportunities for students. MISSEs 3 and 4 have transported approximately 8 million basil seeds for science experiments of children to stimulate interest in space science.

A suitcase shaped rugged box known as Passive Experiment Containers (PECs) is used to transport the selected materials to and from the ISS. PECs were originally developed and used by NASA's LaRC, Hampton, Virginia, for ISS

* narasimha.s.prasad@nasa.gov; Phone 757-864-9403; Fax 757-864-8828. 
Phase I Risk Mitigation Program experiments conducted on the Russian Mir space station. Specific steps required for transporting materials include specimen preparation, fixing specimens inside a holder, integration of specimen holder on a tray, integration of trays in a PEC and integration of PEC inside a carrier for ease of transport. All these tasks are carried out in clean room environment.

The PEC is tested for survivability under launch conditions by subjecting it to appropriate shock and vibration environment. Subsequent to several months of space exposure, the PEC will be transported back to the Earth to undergo tests. The resulting test characteristics when compared with original characteristics before launch will provide an understanding of their survivability in space conditions. Furthermore, it will provide insights into required space qualification processes for future space applications. Terrestrial laboratories may provide limited environmental test conditions while in space, the components are simultaneously exposed to several conditions which normally are difficult to simulate. In many cases, the combined effect of being exposed to all of the environmental elements at once would provide insights into ruggedization for future space applications.

Two possible mounting positions known as ram and wake are available. The materials in the ram side will be predominantly subjected to atomic oxygen environment where as those materials in the wake side will mostly undergo UV exposure. During space walk, also known as extra-vehicular activity (EVA) (i.e., when an astronaut works outside of a spacecraft), the PECs are attached to the handrails or at a specific location that is exposed to space environment. After exposure in space, MISSE PEC is retrieved in the same manner as it was deployed. MISSE PECs have active and passive detectors to give a time-history reading, or a reading of what happened to the materials at certain points in time. The passive detectors report a cumulative measure of the following environments that the test specimens are exposed to namely UV exposure, atomic oxygen exposure, molecular contamination, tray temperature and man-made debris. Back on the ground, tests will be conducted to determine the effects of its exposure for several months in space. The material samples are tested in laboratory conditions to see if they still possess their unique properties needed to complete space missions. The details of MISSE 1 to MISSE 5 missions can be found at NASA's MISSE webpage ${ }^{2}$. In this paper, an overview of MISSE 6 mission followed by optical components for lidar applications packaged by NASA LaRC for MISSE 7 mission is discussed.

\section{SELECTED ITEMS FOR MISSE 6 MISSION}

At NASA LaRC, Laser Remote Sensing Branch in Systems Engineering Directorate (SED) is involved in research and development of novel technologies including space based lidars suitable for future space exploration missions. Among several specimens from NASA Langley, various optical and laser related components were included in the MISSE 6 mission. MISSE 6 package has two sections namely MISSE 6A and MISSE 6B corresponding to ram and wake sides, respectively. The components from SED were located on the MISSE 6B section. In the following sections, a brief summary of these specimens are discussed.

\subsection{Optical elements for MISSE 6 mission}

Table 1 lists novel materials and components that are of interest to NASA.. Following characterization in the laboratory conditions, these items were assembled on the MISSE 6B platform specifically for UV and radiation exposure tests. The objective is to evaluate the impact of space elements on these items for long term operational stability and survivability in space conditions for risk mitigation purposes. The data gathered under MISSE 6 mission will play a significant role in further development of these items for space applications. A brief description on significance of items listed in Table 1 is as follows:

Table 1. Optical specimens and their specifications

\begin{tabular}{|c|c|c|c|c|c|}
\hline No. & TEnO & 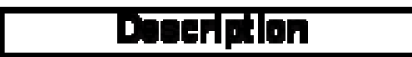 & Emenulone & Unlt: & r.men \\
\hline 1 & $\begin{array}{l}\text { Caronk Yuh } \\
\text { (mas) }\end{array}$ & 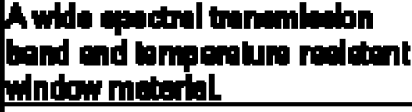 & 12xifod & $\mathbf{m} \mathbf{m i}^{3}$ & Poll:tas \\
\hline 2 & EPbTo & 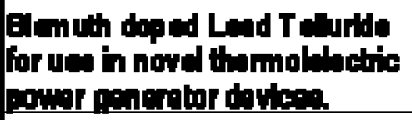 & 12,1206 & $\min$ & Polloted oplod matol \\
\hline 3 & |ctodn & TO-3 Pucleate & $00 \times 25$ & $\min { }^{2}$ & orgns im h ilamote \\
\hline 4 & Morrerulta Lar & Leor axh componato & $\operatorname{ber} 0 \mathrm{n} 2$ & $\min ^{2}$ & trilliph \\
\hline
\end{tabular}


Ceramic Yttria: Ceramic Yttria has been developed for novel UV laser applications under recently completed NASA SBIR Phase II program. In this program, optical quality Nd ion doped ceramic Yttria material of 1" in diameter has been successfully developed using unique material processing techniques ${ }^{4}$. Up to 2" diameter un-doped ceramic Yttria disks have been developed. Yttria has spectral transmission range starting from $200 \mathrm{~nm}$. Greater than $99 \%$ transmission coefficient over visible and near-IR spectral band has been measured. This material is anticipated to play a significant role in the development of compact short pulsed high pulse energy lasers for remote sensing of trace gases, predominantly ozone, as well as in chem-bio sensing applications. One of the secondary applications of ceramic Yttria is in optical windows for crew launch vehicles (CLVs) and crew exploration vehicles (CEVs). This material has potential for use as windows in high temperature $(>2400 \mathrm{C})$ and high pressure $(>30,000 \mathrm{psi})$ environments. Space crafts will be subjected to these conditions during re-entry into the Earth's atmosphere.

Bismuth doped Lead Telluride (Bi:PbTe) and Silver doped Antimony telluride ( $\left.\mathrm{Ag}_{\mathbf{S}} \mathrm{Sb}_{2} \mathrm{Te}_{3}\right)$ : These two semiconductor materials are being investigated for the development of improved efficiency, all solid-state, and compact thermoelectric power generators and cooling elements using nanotechnology. This effort was partially supported under NASA LaRC's funding. Bi:PbTe as n-type and $\mathrm{Ag}: \mathrm{Sb}_{2} \mathrm{Te}_{3}$ as p-type have been successfully tested. So far, promising results have been obtained in regard to their figure-of-merit characteristics. Following material development, fabrication of a prototype thermo electric power generator device has been completed and is now being characterized. Thermoelectric power generators using various heat sources including solar radiation, radioactive isotopes, and waste heat can generate modest electrical power over long durations and hence are attractive for lunar, Mars and other planetary missions. Unlike solar cells, thermoelectric power generators can operate under sealed conditions thereby avoiding damages from extraneous sources.

Semiconductor Laser Diode: The laser diode of interest operates around 2 micron wavelength region. Survivability of bonding and packaging elements are of interest. Besides eyesafe optical communications, this laser diode can be used for trace gas detection specifically Carbon dioxide $\left(\mathrm{CO}_{2}\right)$ species. Coupled with MOEMS architecture, miniature and cost effective $\mathrm{CO}_{2}$ sensors can be developed using this laser diode technology for use in localized environments, short range Differential lidar applications as well as static sensors for tracking gas clouds on Martian environment. Static sensors could be configured as distributed point sensors based network on Martian environment.

Waveguide Laser Components: Waveguide laser for operation at $1064 \mathrm{~nm}$ wavelength include an high power $808 \mathrm{~nm}$ pump diode bar, laser gain medium, a saturable absorber, and dielectric mirrors. Waveguide laser is an emerging technology that offers several unique features. Compared to other solid-state laser technologies, such as fiber lasers, the self-imaging waveguide offers higher peak and average power performance, and superior thermal waste heat management. As such, they are attractive as reliable, stable sources for imaging and high bit rate, high power optical communications. The unique, patented waveguide gain media ${ }^{5}$ offers additional capabilities for space applications, such as amplifiers and pump sources for nonlinear optical devices. The laser diodes will provide risk reduction data for producing higher mean time between failure (MTBF) devices. The optical components that are proposed for space exposure testing are relevant to laser communication, laser range finding, hyperspectral imaging, and vibrometry applications. Furthermore, waveguide based lasers would be suitable for expanding future space communication networks, proximity operations, and guidance, navigation and control.

\subsection{Optical elements on a specimen holder}

Figure 1 shows the optical items mounted on a specimen holder. The laser diode, diode bar, ceramic yttria and Bismuth doped $\mathrm{PbTe}$ are visible. Other elements are below a secondary plate and are hidden from direct view. This specimen holder was mounted on MISSE 6B. Figure 2 shows PEC mounted on a carrier with all other specimen trays. Figure 3 illustrates fully integrated PEC on a test platform. 

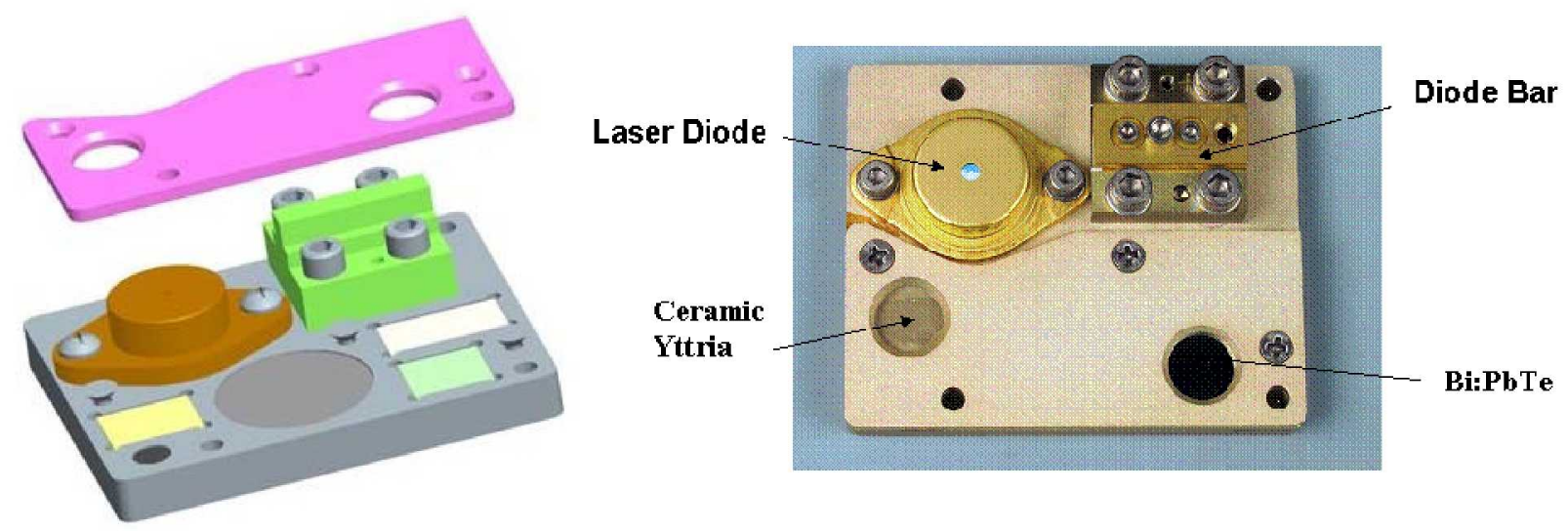

Fig. 1. The optical elements rigidly mounted in a specimen holder.

Fig. 2. The specimen holder is integrated inside the PEC with carrier. The figure insert on top right shows the location of our specimen holder.

Fig. 3. The PEC with carrier on a test platform.

\section{DEPLOYMENT OF MISSE 6}

The MISSE 6 PEC was transported on STS-123 Mission to ISS on March 11, 2008. On Tuesday, March 18, 2008, during the mission's third spacewalk, the Mission Specialist Rick Linnehan attempted to install the MISSE 6 experiment onto the Columbus module. This attempt failed because latching pins used to hold the experiment packages onto the hull of Columbus could not be properly engaged ${ }^{5}$. Following five spacewalks, astronauts Robert L. Behnken and Mike Foreman completed the final spacewalk of the STS-123 mission at 9:36 p.m. CDT. Their 6-hour, 2-minute EVA was highlighted by the installation of the MISSE- 6 . Behnken installed the MISSE- 6 on the outside of the Columbus laboratory. With the help of a few troubleshooting methods developed by engineers on the ground, Behnken successfully installed MISSE- 6 onto the Columbus module ${ }^{6}$. Figure 4 shows the MISSE 6 PEC attached to the Columbus module on the ISS. Figure 5 illustrates the orientation of MISSE 6 along with the velocity vector as seen from the STS-123. 


\section{RETRIEVAL OF MISSE 6 PEC}

The MISSE 6 PEC is anticipated to be retrieved by STS-128 mission scheduled to be launched in August 2009. Following laboratory tests, a detailed comparative analysis of the above mentioned specimens will be carried out to understand space effects. The results will be reported in upcoming conferences.

Fig. 4. (Left) The PEC attached to the Columbus module on ISS. (Right) A close-up view of MISSE PEC in space.

\section{SELECTED ITEMS FOR MISSE 7 MISSION}

MISSE-7 is a suite of experiments that include over 700 new and affordable materials. The selected MISSE 7 specimens have potential use in advanced systems for space applications. For MISSE-7 there are two PECs, 7A and 7B, which will be mounted on the outside of the ISS and hold samples on both sides of the PECs. PEC 7A's orientation will be zenith/nadir (space facing/Earth facing) while PEC 7B will face ram/wake (forward/backward) relative to the ISS orbit. MISSE-7 will be mounted to the ISS's exterior on an EXPRESS Logistics Carrier (ELC). Power and data provided by the ISS will use the ISS communication system uplink/downlink capabilities to receive commands downlink data. No crew interaction is required.

Fig. 5. Orientation of MISSE 6 PEC on ISS.

MISSE 7 is being managed by Glenn Research Center. The MISSE 7 efforts were accelerated in 2008. Items from various research groups were sent to Naval Research Laboratory (NRL), Washington, DC for platform integration and testing. Currently, MISSE 7 is planned to be launched in August 2009 via STS 129 mission. During EVA astronauts will install the MISSE-7 on the ISS. MISSE-7 package will be retrieved at a later date and the specimens will returned to the investigators for testing and post analysis. MISSE 7 could be the last of MISSE missions

NASA LaRC decided to test several lidar transceiver materials for operation in near-IR spectral band. These components include 1.5 micron fiber laser, high power $808 \mathrm{~nm}$ laser diode bar, fiber coupled distributed feedback (DFB) laser, tellurium dioxide $\left(\mathrm{TeO}_{2}\right)$ based acousto-optic modulator and tunable filter, coherent receivers, and several types of acousto-optic and laser gain media including mercurous chloride, mercurous bromide, gallium phosphide, chromium doped zinc selenide (Cr:ZnSe), erbium doped ytterbium aluminum garnet (Er:YAG) and erbium doped lutetium aluminum garnet (Er:LuAG) crystals. Figure 6 shows the laboratory tested components packed on a flight qualified platform. This package will be mounted on MISSE 7 PEC facing wake side investigations primarily for UV and radiation exposure effects. Figure 7 shows platform integration and testing being done at $\mathrm{NRL}^{7}$.

\section{SUMMARY AND CONCLUSIONS}

The objective of the MISSE program is to study the performance, stability, and long-term survivability of novel materials when subjected to the synergistic effects of the harsh space environment. So far, five MISSE missions have been completed. The MISSE 6 mission was launched on March 11, 2008. Several optical specimens including waveguide laser components, laser diodes, thermoelectric power generator materials and ceramic yttria specimens were successfully placed on the ISS for primarily radiation and UV exposure. These specimens will be retrieved in 2009 via STS 128 mission. Several specimens and devices for use in Laser and Lidar systems have been selected from NASA LaRC for MISSE 7 mission. MISSE 7 PEC will be transported to ISS by STS 129 mission. Upon retrieval, these specimens will undergo similar tests to look into any possible deviations from pre-exposure characteristics.

Fig. 6. Orientation of MISSE 6 PEC on ISS. 
Fig. 7. PEC integration and testing of MISSE 7 PEC.

\section{ACKNOWLEDGEMENTS}

The author acknowledges William H. Kinard for providing guidance and support in the initial stages of MISSE 7 mission development. The author is grateful to William C. Edwards for arranging funds for this effort. Finally, the author thanks Karen Gibson and Terry Clark for mechanical engineering and flight package quality control efforts.

\section{REFERENCES}

[1] William H. Kinard, "MIR Environmental Effects Payload (MEEP) Archive System," NASA, Langley Research Center, Hampton, Virginia. (http://setas-www.larc.nasa.gov/meep/meep.html and http://www.nasa.gov/centers/langley/news/factsheets/misse_2005.html)

[2] The NASA MISSE website: http://misseone.larc.nasa.gov/

[3] Prasad, N.S.; Edwards, W.C.; Trivedi, S.B.; Kutcher, S.W.; Chen-Chia Wang; Joo-Soo Kim; Hommerich, U.; Shukla, V.; Sadangi, R.; Kear, B.H., "Recent Progress in the Development of Neodymium-Doped Ceramic Yttria," Selected Topics in IEEE Journal of Quantum Electronics, Vol. 13, Issue 3, 831 - 837(2007).

[4] US Patent Number 6,894,828, "Power scalable waveguide amplifier and laser device"(2005).

[5] STS-123 MCC Status Report \#15 (http://www.nasa.gov/mission_pages/shuttle/shuttlemissions/sts123/news/STS123-15.html).

[6] STS-123 MCC Status Report \#25 (http://www.nasa.gov/mission_pages/shuttle/shuttlemissions/sts123/news/STS123-25.html).

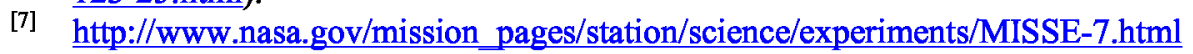

